\title{
INTERVAL LINGUISTIC FUZZY DECISION MAKING IN PERSPECTIVE OF PREFERENCE RELATIONS
}

\author{
Fanyong $\mathrm{MENG}^{1,2^{\star}}$, Jia TANG ${ }^{1}$, Shaolin $\mathrm{ZHANG}^{1}$ \\ ${ }^{1}$ School of Business, Central South University, Changsha, Hunan 410083, China \\ ${ }^{2}$ School of Management and Economics, Nanjing University of Information Science \\ and Technology, Nanjing, Jiangsu 210044, China
}

Received 10 September 2018; accepted 20 April 2019

\begin{abstract}
Consistency analysis is a crucial topic for preference relations. This paper studies the consistency of interval linguistic fuzzy preference relations (ILFPRs) using the constrained interval linguistic arithmetic and introduces a new consistency definition. Then, several properties of this definition are researched. Meanwhile, the connection between this concept and a previous one is discussed. Following this concept, programming models for judging the consistency and for deriving consistent ILFPRs are constructed, respectively. Considering the case that incomplete ILFPRs may be obtained, a programming model for obtaining missing judgments following the consistency discussion is built. Afterwards, the consensus for group decision making (GDM) is studied and a model for adjusting individual ILFPRs to reach the consensus threshold is established. Consequently, an interactive procedure for GDM with ILFPRs is presented. A practical problem is provided to illustrate the utilization of the new algorithm and comparative discussion is offered.
\end{abstract}

Keywords: GDM, ILFPR, consistency, programming model, constrained interval linguistic arithmetic.

JEL Classification: A14, C02, C61, C71.

\section{Introduction}

To cope with the situation where decision problems can only use qualitative judgements, Zadeh (1975) introduced linguistic variables such as "good", "bad", and "fair". To facilitate the application of linguistic variables, Herrera and Herrera-Viedma (2000) presented linguistic term sets (LTSs) to denote them. Considering the calculation of linguistic variables, Herrera, Herrera-Viedma, and Verdegay (1996) introduced the 2-tuple linguistic representation method (TLRM) and Xu (2004a) defined several operations on linguistic variables in the continuous LTSs. It is noticeable that the two methods can avoid information loss in the procedure of calculation. Since then, the theory and application of linguistic decision mak-

${ }^{*}$ Corresponding author. E-mail: mengfanyongtjie@163.com

This is an Open Access article distributed under the terms of the Creative Commons Attribution License (http://creativecommons. org/licenses/by/4.0/), which permits unrestricted use, distribution, and reproduction in any medium, provided the original author and source are credited. 
ing have been received considerable attention. On the other hand, preference relation is an efficient and powerful decision-making tool, which only needs decision makers (DMs) to compare two objects at a time. Since Saaty (1977) introduced preference relation in 1977, it is still one of the most powerful decision-making tools.

Taking the advantages of preference relations and linguistic variables, Herrera and Herrera-Viedma (2000) proposed additive linguistic preference relations (ALPRs) and researched the application using the linguistic choice function and two linguistic choice mechanisms. $\mathrm{Xu}$ (2004a) formally presented the concepts of ALPRs and multiplicative linguistic preference relations (MLPRs), where MLPRs are defined on the asymmetrical linguistic term set, and ALPRs employ linguistic variables in the symmetrical LTS. Then, the author introduced two methods for GDM with MLPRs and ALPRs using the defined linguistic aggregation operators. Notably, Xu (2004a) adopted the multiplicative and additive transitivity to give the concepts of consistent MLPRs and ALPRs, respectively. According to Xu (2004a, 2004b) introduced a method for GDM with MLPRs following the linguistic averaging operators and $\mathrm{Xu}$ (2005) researched ALPRs following the defined deviation and similarity degrees. On the other hand, Wang and Chen (2008) used ALPRs to study multi-criteria decision problems with triangular fuzzy preference relations. To rank objects reasonably, $\mathrm{Xu}, \mathrm{Wu}$, and Wang (2017) offered a method for decision making with ALPRs based on the Gower plot and the ordinal additive consistency and Jin et al. (2017) proposed two new methods for GDM with MLPRs following the acceptably multiplicative consistency. Moreover, incomplete linguistic preference relations (LPRs) are researched in the literature (Alonso, Cabrerizo, Chiclana, Herrera, \& Herrera-Viedma, 2009; Xu, 2006a), while GDM with multi-granular LPRs is discussed in the literature (Herrera-Viedma, Martinez, Mata, \& Chiclana, 2005; Xu, 2008).

The limitation of MLPRs and ALPRs is that DMs can only use an exact linguistic variable to denote one judgement, which cannot express the qualitative uncertainty of DMs' recognitions. To address this problem, $\mathrm{Xu}$ (2004c) presented interval linguistic variables (ILVs) and studied the application in decision making. Following the original work of $\mathrm{Xu}$ (2004c), many additive interval linguistic aggregation operators are proposed (Xu, 2006b; Chen \& Lee, 2012; Park, Gwak, \& Kwun, 2011; Meng, Tan, \& Zhang, 2014; Meng \& Chen, 2015). Furthermore, Tapia García, del Moral, Martinez, and Herrera-Viedma (2012) introduced additive interval linguistic variables to preference relations and presented interval linguistic fuzzy preference relations (ILFPRs). After that, a GDM method based on the consensus measure and the proximity measure is presented. Xu and Wu (2013) used the TLRM to study GDM with ILFPRs based on the consensus analysis and Chen, Zhou, and Han (2011) presented a method for GDM with ILFPRs based on the compatibility degree. Furthermore, Büyüközkan and Güleryüz (2014) studied the application of ILFPRs in renewable energy planning. However, all of these methods did not consider the consistency of ILFPRs that may lead to the illogical ranking. Considering this issue, Meng, Tang, and Xu (2019) studied the consistency of ILFPRs and presented a consistency concept. Following the consistency analysis, the authors introduced a method for GDM with ILFPRs that can solve inconsistent-incomplete ILFPRs. Similar to ILFPRs, multiplicative interval linguistic fuzzy preference relations (MILFPRs) are researched in the literature (Tang, Meng, Li, \& Li, 2018; Xu, 2006c; Zhou, He, Chen, \& Liu, 2014). 
In the procedure of computing the priority weights logically, consistency analysis is the most important and fundamental topic. After recalling previous methods for decision making with ILFPRs, we find that there is only one study on the consistency of ILFPRs (Meng et al., 2019). Although Meng's et al. concept owns all desirable properties of ALPRs (Xu, 2004a), this concept defines on the endpoints of ILVs and requires ALPRs obtained from the endpoints of ILVs to satisfy the additive transitivity. This issue is that there is no argument to define the consistency of ILFPRs by restricting to the endpoints of all ILVs. This requirement seems to be a little restriction. Considering this problem, this paper continues to study the consistency of ILFPRs. To do this, we extend Lodwick and Jenkins's constrained interval arithmetic (Lodwick \& Jenkins, 2013) to ILVs and introduce the constrained interval linguistic arithmetic. Following this, a new consistency concept for ILFPRs is presented and several properties are discussed. Meanwhile, the connection between the new definition and Meng's et al. concept is studied. After that, inconsistent and incomplete ILFPRs are researched using the built programming models. Furthermore, the consensus for GDM with ILFPRs is studied and a consensus measure is presented. Following the consistency-consensus discussion, a new algorithm for GDM with ILFPRs is provided.

The rest of this paper is organized as follows: Section 1 reviews several related definitions including linguistic variables, ILVs, and ILFPRs. Then, Meng's et al. consistency definition is offered and the limitation is analyzed. Section 2 defines the constrained interval linguistic arithmetic and offers a new consistency concept. Meanwhile, it researches the properties of the new concept and discusses the relationship between two consistency concepts. Section 3 focuses on studying inconsistent and incomplete ILFPRs. To rank objects logically, programming models for ascertaining missing values and for obtaining consistent ILFPRs are constructed, respectively. Section 4 mainly researches the consensus and builds a programming model for researching the consensus threshold. Meanwhile, a new algorithm for GDM with ILFPRs is presented. Section 5 offers an example to illustrate the utilization of the new method and comparison discussion of theory and application between the new method and Meng's et al. approach is made. Conclusion is offered in the last Section.

\section{Basic concepts}

Considering the situation where quantitative variables are insufficient to denote the judgments of DMs, Zadeh (1975) introduced linguistic variables. To facilitate the application of linguistic variables, Herrera et al. (1996) introduced the following concept of LTSs:

Definition 1 (Herrera et al., 1996). $S=\left\{s_{i} \mid i=0,1, \ldots, 2 t\right\}$ is called a LTS if the following three conditions

(i) $s_{i}>s_{j}$, if $i>j$,

(ii) $\max \left(s_{i}, s_{j}\right)=s_{i}, \min \left(s_{i}, s_{j}\right)=s_{j}$, if $s_{i} \geq s_{j}$,

(iii) $\operatorname{Neg}\left(s_{i}\right)=s_{2 t-i}$

are true, where $s_{i}$ is a possible value for a linguistic variable, and $t$ is a positive integer.

Later, $\mathrm{Xu}$ (2004a) defined the continuous linguistic term set $\bar{S}=\left\{s_{\alpha} \mid \alpha \in[0,2 t]\right\}$ to avoid information loss. Furthermore, Xu (2004a) defined two operations: 
(i) $s_{\alpha} \oplus s_{\beta}=s_{\alpha+\beta}$,

(ii) $\lambda s_{\alpha}=s_{\lambda \alpha}, \lambda \in[0,1]$

where $s_{\alpha}, s_{\beta} \in \bar{S}$.

To denote the qualitative uncertainty of DMs, Xu (2004c) extended linguistic variables to introduce interval linguistic variables (ILVs): $\bar{s}=\left[s_{\alpha}, s_{\beta}\right]$ is called an ILV if $s_{\alpha} \leq s_{\beta}$ with $s_{\alpha}, s_{\beta} \in \bar{S}$.

Definition $2(\mathrm{Xu}, 2004 \mathrm{c})$. Let $\bar{s}=\left[s_{\alpha}, s_{\beta}\right], \overline{s_{1}}=\left[s_{\alpha_{1}}, s_{\beta_{1}}\right]$, and $\overline{s_{2}}=\left[s_{\alpha_{2}}, s_{\beta_{2}}\right]$ be any three ILVs. Their operational laws are defined as follows:

(i) $\overline{s_{1}} \oplus \overline{s_{2}}=\left[s_{\alpha_{1}+\alpha_{2}}, s_{\beta_{1}+\beta_{2}}\right]$;

(ii) $\overline{s_{1}} \ominus \bar{s}_{2}=\left[s_{\alpha_{1}-\alpha_{2}}, s_{\beta_{1}-\beta_{2}}\right]$;

(iii) $\lambda \bar{s}=\left[s_{\lambda \alpha}, s_{\lambda \beta}\right] \quad \lambda \in[0,1]$;

(iv) $\lambda\left(\overline{s_{1}} \oplus \overline{s_{2}}\right)=\lambda \overline{s_{1}} \oplus \lambda \overline{s_{2}} \quad \lambda \in[0,1]$;

(v) $\left(\lambda_{1}+\lambda_{2}\right) \bar{s}=\lambda_{1} \bar{s} \oplus \lambda_{2} \bar{s} \quad \lambda_{1}, \lambda_{2} \in[0,1]$.

Furthermore, we offer two other operations that will be used in the next section.

(vi) $\min \left\{s_{x} \oplus s_{y} \mid s_{x} \in \overline{s_{1}}, s_{y} \in \overline{s_{2}}\right\}=\min _{s_{x} \in \bar{s}_{1}, s_{y} \in \bar{s}_{2}} s_{x} \oplus s_{y}=\min _{s_{x} \in \overline{s_{1}}, s_{y} \in \overline{s_{2}}} s_{x+y}=s_{\alpha_{1}+\alpha_{2}}$;

(vii) $\max \left\{s_{x} \oplus s_{y} \mid s_{x} \in \overline{s_{1}}, s_{y} \in \overline{s_{2}}\right\}=\max _{s_{x} \in \bar{s}_{1}, s_{y} \in \bar{s}_{2}} s_{x} \oplus s_{y}=\max _{s_{x} \in \bar{s}_{1}, s_{y} \in \bar{s}_{2}} s_{x+y}=s_{\beta_{1}+\beta_{2}}$, where $\bar{s}_{1}$ and $\bar{s}_{2}$ as shown in Definition 2 .

On the basis of ILVs, Tapia García et al. (2012) introduced ILFPRs:

Definition 3 (Tapia García et al., 2012). Let $\bar{R}=\left(\bar{r}_{i j}\right)_{n \times n}$ be a matrix on the finite object set $X=\left\{x_{1}, x_{2}, \ldots, x_{n}\right\} . \bar{R}$ is called an ILFPR if the following conditions

$$
\left\{\begin{array}{l}
r_{i j}^{L}+r_{j i}^{U}=r_{i j}^{U}+r_{j i}^{L}=s_{2 t} \\
r_{i i}^{L}=r_{i i}^{U}=s_{t}
\end{array}\right.
$$

are true for all $i, j=1,2, \ldots, n$, where $\bar{r}_{i j}=\left[r_{i j}^{L}, r_{i j}^{U}\right]$ is an ILV denoting the uncertainly qualitative preferred degree of the object $x_{i}$ over $x_{j}$ with $r_{i j}^{L}, r_{i j}^{U} \in \bar{S}$.

Definition 3 shows that an ILFPR $\bar{R}=\left(\bar{r}_{i j}\right)_{n \times n}$ reduces to an ALPR $R=\left(r_{i j}\right)_{n \times n}$ when all ILVs are linguistic variables, namely, $r_{i j}^{L}=r_{i j}^{U}$ for all $i, j=1,2, \ldots, n$.

Considering the consistency of ILFPRs, Meng et al. (2019) defined quasi ILFPRs (QILFPRs):

Definition 4 (Meng et al., 2019). Let $\bar{R}=\left(\bar{r}_{i j}\right)_{n \times n}$ be an ILFPR, and let $\bar{T}=\left(\bar{t}_{i j}\right)_{n \times n}$ be an associated QILFPR, namely, $\left\{\begin{array}{l}\bar{t}_{i j}=\theta_{i j}\left[r_{i j}^{L}, r_{i j}^{U}\right] \oplus\left(1-\theta_{i j}\right)\left[r_{i j}^{U}, r_{i j}^{L}\right] \\ \bar{t}_{j i}=\theta_{i j}\left[r_{j i}^{U}, r_{j i}^{L}\right] \oplus\left(1-\theta_{i j}\right)\left[r_{j i}^{L}, r_{j i}^{U}\right]\end{array}\right.$, where $\theta_{i j}=0$ or 1 for all $i, j=1$, $2, \ldots, n . \bar{T}$ is additively consistent if the following condition

$$
\bar{t}_{i j}=\bar{t}_{i k} \oplus \bar{t}_{k j} \ominus\left[s_{t}, s_{t}\right]
$$

is true for all $i, k, j=1,2, \ldots, n$.

Definition 4 indicates that judgements in QILFPRs satisfy the additive reciprocity. Furthermore, all elements of QILFPRs are derived from associated ILFPRs, Meng et al. (2019) presented the following consistency definition: 
Definition 5 (Meng et al., 2019). Let $\bar{R}=\left(\bar{r}_{i j}\right)_{n \times n}$ be an ILFPR. $\bar{R}$ is additively consistent when there is a consistent QILFPR based on Definition 4.

Notably, Definition 5 is a natural extension of Xu's consistency definition for ALPRs (Xu, 2004a). However, Definition 4 gives the consistency of ILFPRs by considering the endpoints of ILVs. This seems to be a little restriction because there is no argument to require all ILVs to take endpoints simultaneously.

\section{A new consistency concept}

Meng et al. (2019) adopted QILFPRs to define consistent ILFPRs because ILVs in ILFPRs do not satisfy the additive reciprocity. For example, let $\bar{R}=\left(\bar{r}_{i j}\right)_{n \times n}$ be an ILFPR, we usually cannot derive $\bar{r}_{i j} \oplus \bar{r}_{j i} \neq\left[s_{2 t}, s_{2 t}\right]$. However, the ILVs $\bar{r}_{i j}$ and $\bar{r}_{j i}$ have some relationship and they are not independent. As some researchers noted for intervals (Klir \& Yuan, 1998; Lodwick \& Jenkins, 2013), in this situation, it is unreasonable to apply the normal operations on ILVs. Similar to Krejčís constrained interval arithmetic (Krejčí, 2017), we introduce the following

constrained interval linguistic arithmetic for each pair of the ILVs $\left\{\begin{array}{l}\bar{r}_{i j}=\left[r_{i j}^{L}, r_{i j}^{U}\right] \\ \quad \text { Let }\end{array} \bar{r}_{j i}=\left[r_{j i}^{U}, r_{j i}^{L}\right]\right.$

$$
\begin{aligned}
& r_{i j}^{-}=\min \left\{r_{i j} \widetilde{\oplus} r_{j i} \mid r_{i j} \in\left[r_{i j}^{L}, r_{i j}^{U}\right], r_{j i} \in\left[r_{j i}^{L}, r_{j i}^{U}\right], r_{j i}=s_{2 t} \ominus r_{i j}\right\}=s_{2 t} ; \\
& r_{i j}^{+}=\max \left\{r_{i j} \widetilde{\oplus} r_{j i} \mid r_{i j} \in\left[r_{i j}^{L}, r_{i j}^{U}\right], r_{j i} \in\left[r_{j i}^{L}, r_{j i}^{U}\right], r_{j i}=s_{2 t} \ominus r_{i j}\right\}=s_{2 t} .
\end{aligned}
$$

Following formulae (3) and (4), we derive

$$
\left[r_{i j}^{L}, r_{i j}^{U}\right] \widetilde{\oplus}\left[r_{j i}^{U}, r_{j i}^{L}\right]=\left[s_{2 t}, s_{2 t}\right]
$$

for each pair of $(i, j)$ with $i \neq j$.

Different from Meng's et al. consistency definition that is defined on the endpoints of ILVs, we offer a new consistency definition for ILFPRs:

Definition 6. Let $\bar{R}=\left(\bar{r}_{i j}\right)_{n \times n}$ be an ILFPR. $\bar{R}$ is additively consistent if there are $r_{i k} \in \bar{r}_{i k}$ and $r_{k j} \in \bar{r}_{k j}$ such that

$$
r_{i j}=r_{i k} \oplus r_{k j} \ominus s_{t}
$$

for any $r_{i j} \in \bar{r}$ and each triple of $(i, k, j)$.

Remark 1. The main difference between Definitions 4 and 6 is that Definition 6 relaxes the consistency condition for only considering the endpoints of ILVs. In other words, Definition 6 studies the consistency of ILFPRs by considering all possible ALPRs, respectively, while Definition 4 gives the consistency of ILFPRs by considering all possible ALPRs simultaneously.

Next, we discuss the properties of Definition 6, by which one can check that all characteristics of Xu' consistency definition for ALPRs are still true.

Theorem 1. Let $\bar{R}=\left(\bar{r}_{i j}\right)_{n \times n}$ be an ILFPR. Then, the following conclusions are equivalent:

(i) $\bar{R}=\left(\bar{r}_{i j}\right)_{n \times n}$ is consistent according to Definition 6; 
(ii) There are $r_{i k} \in \bar{r}_{i k}$ and $r_{k j} \in \bar{r}_{k j}$ such that $r_{i j}=r_{i k} \oplus r_{k j} \ominus s_{t}$ for any $r_{i j} \in \bar{r}$ and each triple of $(i, k, j)$ with $i<k<j$;

(iii) There are $r_{i k} \in \bar{r}_{i k}$ and $r_{k j} \in \bar{r}_{k j}$ such that $r_{i j} \oplus r_{j k} \oplus r_{k i}=r_{j i} \oplus r_{i k} \oplus r_{k j}$ for any $r_{i j} \in \bar{r}$ and each triple of $(i, k, j)$ with $i<k<j$, where $r_{j i}=s_{2 t} \ominus r_{i j}, r_{j k}=s_{2 t} \ominus r_{k j}$, and $r_{k i}=s_{2 t} \ominus$ $r_{i k}$

(iv) There are $r_{j k} \in \bar{r}_{j k}$ and $r_{k i} \in \bar{r}_{k i}$ such that $r_{i j} \oplus r_{j k} \oplus r_{k i}=s_{3 t}$ for any $r_{i j} \in \bar{r}$ and each triple of $(i, k, j)$ with $i<k<j$;

(v) There are $r_{j k} \in \bar{r}_{j k}$ and $r_{k i} \in \bar{r}_{k i}$ for all $k=1,2, \ldots, n$ such that $r_{i j}=\frac{1}{n} \oplus_{k=1}^{n}\left(r_{i k} \oplus r_{k j}\right) \ominus$ $s_{t}$ for any $r_{i j} \in \bar{r}$ with $i<j$, where $r_{j i}=s_{2 t} \ominus r_{i j}, r_{j k}=s_{2 t} \ominus r_{k j}$, and $r_{k i}=s_{2 t} \ominus r_{i k}$.

Proof. It is obvious that the conclusion (ii) holds under the condition (i). When we have the conclusion (ii), we derive

$$
s_{2 t} \ominus r_{i j}=\left(s_{2 t} \ominus r_{k j}\right) \oplus\left(s_{2 t} \ominus r_{i k}\right) \ominus s_{t} \Rightarrow r_{j i}=r_{j k} \oplus r_{k i} \ominus s_{t} .
$$

Combining formulae (6) and (7), we derive $r_{i j} \oplus r_{j k} \oplus r_{k i}=r_{j i} \oplus r_{i k} \oplus r_{k j}$ for any $r_{i j} \in \bar{r}$ and each triple of $(i, k, j)$ with $i<k<j$. Thus, the conclusion (iii) holds according to the conclusion (ii).

Following the conclusion (iii), we obtain $r_{i j} \oplus r_{j k} \oplus r_{k i}=\left(s_{2 t} \ominus r_{i j}\right) \oplus\left(s_{2 t} \ominus r_{k i}\right) \oplus\left(s_{2 t} \ominus\right.$ $\left.r_{j k}\right)$, by which we have $r_{i j} \oplus r_{j k} \oplus r_{k i}=s_{3 t}$ for any $r_{i j} \in \bar{r}$ and each triple of $(i, k, j)$ with $i<k<j$. Thus, the conclusion (iv) holds.

Under the condition (iv), we get

$r_{i j} \oplus\left(s_{2 t} \ominus r_{i k}\right) \oplus\left(s_{2 t} \ominus r_{k j}\right)=s_{3 t} \Rightarrow r_{i j}=r_{i k} \oplus r_{k j} \ominus s_{t} \Rightarrow n r_{i j}=\oplus_{k=1}^{n}\left(r_{i k} \oplus r_{k j} \ominus s_{t}\right) \Rightarrow$ $r_{i j}=\frac{1}{n} \oplus_{k=1}^{n}\left(r_{i k} \oplus r_{k j}\right) \ominus s_{t}$. Thus,

With the condition (v), we obtain $r_{i k}=\frac{1}{n} \oplus_{l=1}^{n}\left(r_{i l} \oplus r_{l k}\right) \ominus s_{t}$ and $r_{k j}=\frac{1}{n} \oplus_{l=1}^{n}\left(r_{k l} \oplus r_{l j}\right) \ominus s_{t}$.

$$
\begin{aligned}
& r_{i k} \oplus r_{k j}=\left(\frac{1}{n} \oplus_{l=1}^{n}\left(r_{i l} \oplus r_{l k}\right) \ominus s_{t}\right) \oplus\left(\frac{1}{n} \oplus_{l=1}^{n}\left(r_{k l} \oplus r_{l j}\right) \ominus s_{t}\right)= \\
& \left(\frac{1}{n} \oplus_{l=1}^{n}\left(r_{i l} \oplus r_{l k} \oplus r_{k l} \oplus r_{l j}\right)\right) \ominus s_{2 t}=\left(\frac{1}{n} \oplus_{l=1}^{n}\left(r_{i l} \oplus r_{l j}\right) \oplus s_{2 t}\right) \ominus s_{2 t}=\frac{1}{n} \oplus_{l=1}^{n}\left(r_{i l} \oplus r_{l j}\right),
\end{aligned}
$$

by which we have $r_{i j}=r_{i k} \oplus r_{k j} \ominus s_{t}$ for any $r_{i j} \in \bar{r}$ and each triple of $(i, k, j)$ with $i<k<j$. Following $r_{j i}=s_{2 t} \ominus r_{i j}, r_{j k}=s_{2 t} \ominus r_{k j}$, and $r_{k i}=s_{2 t} \ominus r_{i k}$, we further derive $s_{2 t} \ominus r_{i j}=\left(s_{2 t} \ominus r_{i k}\right) \oplus$ $\left(s_{2 t} \ominus r_{k j}\right) \ominus s_{t}$, namely, $r_{j i}=r_{j k} \oplus r_{k i} \ominus s_{t}$. Therefore, we obtain the conclusion (i).

Theorem 2. Let $\bar{R}=\left(\bar{r}_{i j}\right)_{n \times n}$ be an ILFPR. Definition 6 is robust to the compared orders of objects.

Proof. In general, let $i<k<j$. Let $\sigma$ be a permutation on $\{1,2, \ldots, n\}$, where $\sigma(i)=j, \sigma(k)=i$, and $\sigma(j)=k$. Then,

$$
\bar{r}_{\sigma(i) \sigma(j)}=\bar{r}_{j k}, \bar{r}_{\sigma(k) \sigma(j)}=\bar{r}_{i k}, \bar{r}_{\sigma(i) \sigma(k)}=\bar{r}_{j i} .
$$

Following formula (6), we know that there are $r_{j i}=r_{\sigma(i) \sigma(k)} \in \bar{r}_{\sigma(i) \sigma(k)}=\bar{r}_{j i}$ and $r_{i k}=r_{\sigma(k) \sigma(j)} \in \bar{r}_{\sigma(k) \sigma(j)}=\bar{r}_{i k}$ such that

$$
r_{\sigma(i) \sigma(j)}=r_{\sigma(i) \sigma(k)} \oplus r_{\sigma(k) \sigma(j)} \ominus s_{t},
$$

namely, 
$r_{j k}=r_{j i} \oplus r_{i k} \ominus s_{t}$

for any $r_{j k}=r_{\sigma(i) \sigma(j)} \in \bar{r}_{\sigma(i) \sigma(j)}=\bar{r}_{j k}$ and each triple of $(i, k, j)$.

Thus, $\bar{R}^{\sigma}=\left(\bar{r}_{\sigma(i) \sigma(j)}\right)_{n \times n}$ is consistent following Definition 6 .

Because there are infinite cases for judging the consistency of ILFPRs by direct using formula (6), we consider two other equivalent conditions.

Theorem 3. Let $\bar{R}=\left(\bar{r}_{i j}\right)_{n \times n}$ be an ILFPR. It is consistent if and only if the following conditions

$$
r_{i j}^{L} \geq r_{i k}^{L} \oplus r_{k j}^{L} \ominus s_{t} \text { and } r_{i j}^{U} \leq r_{i k}^{U} \oplus r_{k j}^{U} \ominus s_{t}
$$

are true for each triple of $(i, k, j)$.

Proof. When $\bar{R}$ is consistent according to Definition 6, we have $r_{i k} \in \bar{r}_{i k}$ and $r_{k j} \in \bar{r}_{k j}$ such that $r_{i j}^{L}=r_{i k} \oplus r_{k j} \ominus s_{t}$

for $r_{i j}^{L}$ and each triple of $(i, k, j)$. From $r_{i k} \geq r_{i k}^{L}$ and $r_{k j} \geq r_{k j}^{L}$, we derive $r_{i j}^{L} \geq r_{i k}^{L} \oplus r_{k j}^{L} \ominus s_{t}$. Furthermore, we obtain $r_{i k} \in \bar{r}_{i k}$ and $r_{k j} \in \bar{r}_{k j}$ such that

$$
r_{i j}^{U}=r_{i k} \oplus r_{k j} \ominus s_{t}
$$

for $r_{i j}^{U}$ and each triple of $(i, k, j)$. Thus, $r_{i j}^{U} \leq r_{i k}^{U} \oplus r_{k j}^{U} \ominus s_{t}$ based on $r_{i k} \leq r_{i k}^{U} \wedge r_{k j} \leq r_{k j}^{U}$.

On the other hand, when formula (8) holds, we have $\left[r_{i j}^{L}, r_{i j}^{U}\right] \subseteq\left[r_{i k}^{L} \oplus r_{k j}^{L} \ominus s_{t}, r_{i k}^{U} \oplus r_{k j}^{U} \ominus\right.$ $s_{t}$ ], which means that formula (6) holds.

Corollary 1. Let $\bar{R}=\left(\bar{r}_{i j}\right)_{n \times n}$ be an ILFPR. It is consistent if and only if the following conditions

$$
r_{i j}^{L} \geq \max _{k=1,2, \ldots, n, k \neq i, j}\left\{r_{i k}^{L} \oplus r_{k j}^{L} \ominus s_{t}\right\} \text { and } r_{i j}^{U} \leq \min _{k=1,2, \ldots, n, k \neq i, j}\left\{r_{i k}^{U} \oplus r_{k j}^{U} \ominus s_{t}\right\}
$$

are true for each pair of $(i, j)$ with $i<j$.

Now, we consider the relationship between Definitions 4 and 6 .

Theorem 4. Let $\bar{R}=\left(\bar{r}_{i j}\right)_{n \times n}$ be an ILFPR. When it is consistent following Definition 6, it is consistent according to Definition 4 . However, the opposite conclusion is not true, that's, if it is consistent following Definition 4, it will not be necessarily consistent following Definition 6.

Proof. When $\bar{R}$ is consistent following Definition 4 , we have

$\theta_{i j}\left[r_{i j}^{L}, r_{i j}^{U}\right] \oplus\left(1-\theta_{i j}\right)\left[r_{i j}^{U}, r_{i j}^{L}\right]=\left(\theta_{i k}\left[r_{i k}^{L}, r_{i k}^{U}\right] \oplus\left(1-\theta_{i k}\right)\left[r_{i k}^{U}, r_{i k}^{L}\right]\right) \oplus\left(\theta_{k j}\left[r_{k j}^{L}, r_{k j}^{U}\right] \oplus\left(1-\theta_{k j}\right)\left[r_{k j}^{U}, r_{k j}^{L}\right]\right) \ominus$ $\left[s_{t}, s_{t}\right]$

for each triple of $(i, k, j)$, by which we obtain

$$
\begin{aligned}
& \theta_{i j} r_{i j}^{L} \oplus\left(1-\theta_{i j}\right) r_{i j}^{U}=\left(\theta_{i k} r_{i k}^{L} \oplus\left(1-\theta_{i k}\right) r_{i k}^{U}\right) \oplus\left(\theta_{k j} r_{k j}^{L} \oplus\left(1-\theta_{k j}\right) r_{k j}^{U}\right) \ominus s_{t} ; \\
& \theta_{i j} r_{i j}^{U} \oplus\left(1-\theta_{i j}\right) r_{i j}^{L}=\left(\theta_{i k} r_{i k}^{U} \oplus\left(1-\theta_{i k}\right) r_{i k}^{L}\right) \oplus\left(\theta_{k j} r_{k j}^{U} \oplus\left(1-\theta_{k j}\right) r_{k j}^{L}\right) \ominus s_{t} .
\end{aligned}
$$

For $\theta_{i j}=1$ or 0 , we both have $r_{i j}^{L} \geq r_{i k}^{L} \oplus r_{k j}^{L} \ominus s_{t}$ and $r_{i j}^{U} \leq r_{i k}^{U} \oplus r_{k j}^{U} \ominus s_{t}$ for each triple of $(i, k, j)$. According to Theorem 3, we conclude that it is consistent based on Definition 6.

To show the converse conclusion, we offer the following example. Let $X=\left\{x_{1}, x_{2}, x_{3}\right\}$, and let the ILFPR $\bar{R}=\left(\bar{r}_{i j}\right)_{3 \times 3}$ on $X$ for the predefined LTS $S=\left\{s_{i} \mid i=1,2, \ldots, 8\right\}$ is defined 
as follows:

$$
\bar{R}=\left(\begin{array}{lll}
{\left[s_{4}, s_{4}\right]} & {\left[s_{4}, s_{6}\right]} & {\left[s_{5}, s_{8}\right]} \\
{\left[s_{2}, s_{4}\right]} & {\left[s_{4}, s_{4}\right]} & {\left[s_{5}, s_{7}\right]} \\
{\left[s_{0}, s_{3}\right]} & {\left[s_{1}, s_{3}\right]} & {\left[s_{4}, s_{4}\right]}
\end{array}\right) .
$$

One can find that $\bar{R}$ is consistent following Definition 6, but it is inconsistent according to Definition 4.

Following Theorem 4, the connections between Definitions 4 and 6 can be intuitively shown in Figure 1.

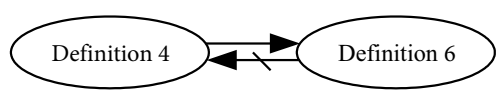

Figure 1. The relationship between Definitions 4 and 6

\section{Inconsistent and incomplete ILFPRs}

For a given ILFPR $\bar{R}=\left(\bar{r}_{i j}\right)_{n \times n}$, we can apply formula (8) to judge its consistency. The issue is that ILFPRs offered by the DMs are usually inconsistent. To derive ranking logically, consistency adjustment is needed. Nevertheless, it is not an easy thing to derive consistent ILFPRs using formula (8) directly. With different adjusted ILVs, ranking values and orders may be different. For example, let the ILFPR $\bar{R}=\left(\bar{r}_{i j}\right)_{3 \times 3}$ on $X=\left\{x_{1}, x_{2}, x_{3}\right\}$ for the predefined LTS $S=$ $\left\{s_{i} \mid i=1,2, \ldots, 8\right\}$ is defined as follows:

$$
\bar{R}=\left(\begin{array}{lll}
{\left[s_{4}, s_{4}\right]} & {\left[s_{3}, s_{5}\right]} & {\left[s_{5}, s_{6}\right]} \\
{\left[s_{3}, s_{5}\right]} & {\left[s_{4}, s_{4}\right]} & {\left[s_{2}, s_{3}\right]} \\
{\left[s_{2}, s_{3}\right]} & {\left[s_{5}, s_{6}\right]} & {\left[s_{4}, s_{4}\right]}
\end{array}\right) .
$$

From $r_{12}^{L}=s_{3}<r_{13}^{L}+r_{32}^{L}=s_{5} \oplus s_{5} \ominus s_{4}$, we know that $\bar{R}$ is inconsistent based on Definition 6 . In this case, we need to adjust some interval linguistic judgements to obtain consistent ILFPR.

If we adjust the ILVs $\bar{r}_{12}$ and $\bar{r}_{21}$, by the minimum adjustment we obtain $\bar{r}_{12}^{\prime}=\left[s_{6}, s_{7}\right]$ and $\bar{r}_{21}^{\prime}=\left[s_{1}, s_{2}\right]$ for obtaining the consistent ILFPR, where

$$
\bar{R}_{1}=\left(\begin{array}{lll}
{\left[s_{4}, s_{4}\right]} & {\left[s_{6}, s_{7}\right]} & {\left[s_{5}, s_{6}\right]} \\
{\left[s_{1}, s_{2}\right]} & {\left[s_{4}, s_{4}\right]} & {\left[s_{2}, s_{3}\right]} \\
{\left[s_{2}, s_{3}\right]} & {\left[s_{5}, s_{6}\right]} & {\left[s_{4}, s_{4}\right]}
\end{array}\right) .
$$

When we only adjust the ILVs $\bar{r}_{13}$ and $\bar{r}_{31}$, by the minimum adjustment we have $\bar{r}_{13}^{\prime}=\left[s_{2}, s_{3}\right]$ and $\bar{r}_{31}^{\prime}=\left[s_{5}, s_{6}\right]$ for obtaining the consistent ILFPR, where

$$
\bar{R}_{2}=\left(\begin{array}{lll}
{\left[s_{4}, s_{4}\right]} & {\left[s_{3}, s_{5}\right]} & {\left[s_{2}, s_{3}\right]} \\
{\left[s_{3}, s_{5}\right]} & {\left[s_{4}, s_{4}\right]} & {\left[s_{2}, s_{3}\right]} \\
{\left[s_{2}, s_{3}\right]} & {\left[s_{5}, s_{6}\right]} & {\left[s_{4}, s_{4}\right]}
\end{array}\right) .
$$

Furthermore, if we only adjust the ILVs $\bar{r}_{23}$ and $\bar{r}_{32}$, by the minimum adjustment we derive $\bar{r}_{23}{ }_{23}=\left[s_{4}, s_{6}\right]$ and $\bar{r}^{\prime}{ }_{31}=\left[s_{2}, s_{4}\right]$ for obtaining the consistent ILFPR, where

$$
\bar{R}_{3}=\left(\begin{array}{lll}
{\left[s_{4}, s_{4}\right]} & {\left[s_{3}, s_{5}\right]} & {\left[s_{5}, s_{6}\right]} \\
{\left[s_{3}, s_{5}\right]} & {\left[s_{4}, s_{4}\right]} & {\left[s_{4}, s_{6}\right]} \\
{\left[s_{2}, s_{3}\right]} & {\left[s_{2}, s_{4}\right]} & {\left[s_{4}, s_{4}\right]}
\end{array}\right) .
$$


With respect to these three cases, using the arithmetic average value of each row (Meng et al., 2019), the interval linguistic priority weight vectors are derived as follows:

$$
\begin{aligned}
& \bar{W}_{1}=\left(\left[s_{5}, s_{\frac{17}{3}}\right],\left[s_{\frac{7}{3}}, s_{3}\right],\left[s_{\frac{11}{3}}, s_{\frac{13}{3}}\right]\right), \bar{W}_{2}=\left(\left[s_{3}, s_{4}\right],\left[s_{3}, s_{4}\right],\left[s_{\frac{11}{3}}, s_{\frac{13}{3}}\right]\right) \text { and } \\
& \bar{W}_{3}=\left(\left[s_{4}, s_{5}\right],\left[s_{\frac{11}{3}}, s_{5}\right],\left[s_{\frac{8}{3}}, s_{\frac{11}{3}}\right]\right) .
\end{aligned}
$$
Following the formula for ranking ILVs in (Meng, An, \& Chen, 2016), we get $\left\{\begin{array}{l}x_{1} \succ x_{3} \succ x_{2} \\ x_{3} \succ x_{1}=x_{2} \\ x_{1} \succ x_{2} \succ x_{3}\end{array}\right.$,
respectively.

Thus, it is insufficient to only apply formula (8) to judge the consistency of ILFPRs. Next, we build two programming models to derive consistent ILFPRs with the minimum adjustment.

For any $s_{\alpha} \in \bar{S}$, let $I\left(s_{\alpha}\right)=\alpha$. Let $\bar{R}=\left(\bar{r}_{i j}\right)_{n \times n}$ be the given ILFPR. To judge its consistency, we build the following programming model:

$$
\begin{aligned}
& \phi^{*}=\min \sum_{k=1, k \neq i, j}^{n}\left(\gamma_{i j}^{-}+\gamma_{i j}^{+}\right) \\
& \text {s.t. }\left\{\begin{array}{l}
I\left(r_{i j}^{L}\right)+\gamma_{i j}^{-} \geq I\left(r_{i k}^{L}\right)+I\left(r_{k j}^{L}\right)-t \\
I\left(r_{i j}^{U}\right)-\gamma_{i j}^{+} \leq I\left(r_{i k}^{U}\right)+I\left(r_{k j}^{U}\right)-t \\
k=1,2, \ldots, n, k \neq i, j \\
\gamma_{i j}^{-}, \gamma_{i j}^{+} \geq 0 \\
i, j=1,2, \ldots, n, i<j
\end{array},\right.
\end{aligned}
$$

where the first two constraints are obtained from formula (8) by adding the deviation values $\gamma_{i j}^{-}$and $\gamma_{i j}^{+}$for all $i, j=1,2, \ldots, n$ with $i<j$.

Following model (M-1), if $\phi^{*}=0$, then it is consistent. Otherwise, it is inconsistent. In this situation, we add the deviation variables to formula (8) and build the following programming model:

$$
\begin{aligned}
\varphi^{*}=\min & \sum_{i, j=1, i \neq j}^{n}\left(\alpha_{i j}^{+}+\alpha_{i j}^{-}+\beta_{i j}^{+}+\beta_{i j}^{-}\right) \\
& s . t .\left\{\begin{array}{l}
I\left(r_{i j}^{L}\right)-\alpha_{i j}^{+}+\alpha_{i j}^{-} \geq\left(I\left(r_{i k}^{L}\right)-\alpha_{i k}^{+}+\alpha_{i k}^{-}\right)+\left(I\left(r_{k j}^{L}\right)-\alpha_{k j}^{+}+\alpha_{k j}^{-}\right)-t \\
I\left(r_{i j}^{U}\right)-\beta_{i j}^{+}+\beta_{i j}^{-} \leq\left(I\left(r_{i k}^{U}\right)-\beta_{i k}^{+}+\beta_{i k}^{-}\right)+\left(I\left(r_{k j}^{U}\right)-\beta_{k j}^{+}+\beta_{k j}^{-}\right)-t \\
k=1,2, \ldots, n, k \neq i, j \\
\alpha_{i j}^{-}-\alpha_{i j}^{+}+\beta_{j i}^{-}-\beta_{j i}^{+}=0 \\
\beta_{i j}^{-}-\beta_{i j}^{+}+\alpha_{j i}^{-}-\alpha_{j i}^{+}=0 \\
I\left(r_{i j}^{L}\right)-\alpha_{i j}^{+}+\alpha_{i j}^{-} \leq I\left(r_{i j}^{U}\right)-\beta_{i j}^{+}+\beta_{i j}^{-} \\
0 \leq I\left(r_{i j}^{L}\right)-\alpha_{i j}^{+}+\alpha_{i j}^{-} \\
I\left(r_{i j}^{U}\right)-\beta_{i j}^{+}+\beta_{i j}^{-} \leq 2 t \\
\alpha_{i j}^{+}, \alpha_{i j}^{-}, \beta_{i j}^{+}, \beta_{i j}^{-} \geq 0 \\
i, j=1,2, \ldots, n, i<j
\end{array}\right.
\end{aligned}
$$

where the first two constraints are obtained from formula (8) by adding the deviation values on the endpoints of ILVs, respectively, the third and fourth constraints ensure the adjusted linguistic matrix to be still an ILFPR, and the fifth to seventh constraints are derived from the concept of ILVs. 
Following model (M-2), we derive consistent ILFPR $\bar{R}^{*}=\left(\bar{r}_{i j}^{*}\right)_{n \times n}$, where $\bar{r}_{i j}^{*}=$ $\left[s_{I\left(r_{i j}^{L}\right)-\alpha_{i j}^{*+}+\alpha_{i j}^{*-}}, s_{I\left(r_{i j}^{U}\right)-\beta_{i j}^{*+}+\beta_{i j}^{*-}}\right]$ and $\alpha_{i j}^{*+}, \alpha_{i j}^{*-}, \beta_{i j}^{*+}, \beta_{i j}^{*-}$ are obtained from model (M-2) for all $i, j=1,2, \ldots, n$.

In some situations, incomplete ILFPRs may be obtained because of various reasons. To compute the interval linguistic priority weight vector, missing linguistic judgments should be determined firstly.

Theorem 5. Let $\bar{R}=\left(\bar{r}_{i j}\right)_{n \times n}$ be an ILFPR. If it is consistent following Definition 6, then

$$
(n-2) r_{i j}^{L} \geq \oplus_{k=1, k \neq i, j}^{n}\left(r_{i k}^{L} \oplus r_{k j}^{L} \ominus s_{t}\right) \text { and }(n-2) r_{i j}^{U} \leq \oplus_{k=1, k \neq i, j}^{n}\left(r_{i k}^{U} \oplus r_{k j}^{U} \ominus s_{t}\right)
$$

for each pair of $(i, j)$ with $i<j$.

Proof. Following Theorem 3, the conclusion is obviously true.

Based on formula (10), programming model for ascertaining missing linguistic variables is built as follows:

$$
\begin{aligned}
& \xi^{*}=\min \sum_{i=1}^{n-1} \sum_{j=i+1}^{n}\left(\chi_{i j}^{+}+\chi_{i j}^{-}\right) \\
& \text {s.t. }\left\{\begin{array}{l}
(n-2) I\left(r_{i j}^{L}\right)+\chi_{i j}^{-} \geq \sum_{k=1, k \neq i, j}^{n}\left(I\left(r_{i k}^{L}\right)+I\left(r_{k j}^{L}\right)-t\right) \\
(n-2) I\left(r_{i j}^{U}\right)-\chi_{i j}^{+} \leq \sum_{k=1, k \neq i, j}^{n}\left(I\left(r_{i k}^{U}\right) \oplus I\left(r_{k j}^{U}\right)-t\right) \\
\delta_{i j}^{+}, \delta_{i j}^{-} \geq 0, i, j=1,2, \ldots, n, i<j \\
0 \leq I\left(r_{i j}^{L}\right) \leq I\left(r_{i j}^{U}\right), I\left(r_{j i}^{U}\right)=2 t-I\left(r_{i j}^{L}\right), r_{i j}^{L} \in U^{-} \wedge a_{i j}^{+} \notin U^{+} \\
I\left(r_{i j}^{L}\right) \leq I\left(r_{i j}^{U}\right) \leq 2 t, I\left(r_{j i}^{L}\right)=2 t-I\left(r_{i j}^{U}\right), r_{i j}^{L} \notin U^{-} \wedge a_{i j}^{+} \in U^{+} \\
0 \leq I\left(r_{i j}^{L}\right) \leq I\left(r_{i j}^{U}\right) \leq 2 t, I\left(r_{j i}^{U}\right)=2 t-I\left(r_{i j}^{L}\right), I\left(r_{j i}^{L}\right)=2 t-I\left(r_{i j}^{U}\right), r_{i j}^{L} \in U^{-} \wedge a_{i j}^{+} \in U^{+}
\end{array}\right.
\end{aligned}
$$

where the first two constraints are obtained from formula (10), the third to fifth constraints are derived from the definition of ILVs, $U^{-}=\left\{r_{i j}^{L}\right.$ is missing, $\left.i, j=1,2, \ldots, n \wedge i<j\right\}$ and $U^{+}=\left\{r_{i j}^{+}\right.$is missing, $\left.i, j=1,2, \ldots, n \wedge i<j\right\}$.

Similar analysis for inconsistent ILFPRs, more than one linguistic variable may exist for some missing value that satisfies the consistency condition. To solve this issue, programming model for ascertaining missing judgements is built as follows:

$$
\begin{aligned}
\psi^{*}= & \min \sum_{r_{i j}^{L} \in U^{-} \vee a_{i j}^{+} \in U^{+}} d_{i j} \\
\text { s.t. } & \left\{\begin{array}{l}
(n-2) I\left(r_{i j}^{L}\right)+\chi_{i j}^{-} \geq \sum_{k=1, k \neq i, j}^{n}\left(I\left(r_{i k}^{L}\right)+I\left(r_{k j}^{L}\right)-t\right) \\
(n-2) I\left(r_{i j}^{U}\right)-\chi_{i j}^{+} \leq \sum_{k=1, k \neq i, j}^{n}\left(I\left(r_{i k}^{U}\right) \oplus I\left(r_{k j}^{U}\right)-t\right) \\
\delta_{i j}^{+}, \delta_{i j}^{-} \geq 0, i, j=1,2, \ldots, n, i<j \\
\sum_{i=1}^{n-1} \sum_{j=i+1}^{n}\left(\chi_{i j}^{+}+\chi_{i j}^{-}\right)=\xi^{*} \\
d_{i j}=I\left(r_{i j}^{U}\right)-I\left(r_{i j}^{L}\right), r_{i j}^{L} \in U^{-} \vee a_{i j}^{+} \in U^{+} \\
0 \leq I\left(r_{i j}^{L}\right) \leq I\left(r_{i j}^{U}\right), I\left(r_{j i}^{U}\right)=2 t-I\left(r_{i j}^{L}\right), r_{i j}^{L} \in U^{-} \wedge a_{i j}^{+} \notin U^{+} \\
I\left(r_{i j}^{L}\right) \leq I\left(r_{i j}^{U}\right) \leq 2 t, I\left(r_{j i}^{L}\right)=2 t-I\left(r_{i j}^{U}\right), r_{i j}^{L} \notin U^{-} \wedge a_{i j}^{+} \in U^{+} \\
0 \leq I\left(r_{i j}^{L}\right) \leq I\left(r_{i j}^{U}\right) \leq 2 t, I\left(r_{j i}^{U}\right)=2 t-I\left(r_{i j}^{L}\right), I\left(r_{j i}^{L}\right)=2 t-I\left(r_{i j}^{U}\right), r_{i j}^{L} \in U^{-} \wedge a_{i j}^{+} \in U^{+}
\end{array},\right.
\end{aligned}
$$


where $\xi^{*}$ is derived from model (M-3), $d_{i j}$ is the length of the missing ILV $\bar{r}_{i j}=\left[r_{i j}^{U}, r_{i j}^{L}\right]$, and all other constraints as shown in model (M-3).

Model (M-4) considers that the shorter the length of the missing ILV is, the better the ILV will be. To show the application of the above programming models, we offer the following example.

Example 1. Let $X=\left\{x_{1}, x_{2}, x_{3}, x_{4}\right\}$ be the object set, and let $S=\left\{s_{1}, s_{2}, \ldots, s_{8}\right\}$ be the predefined LTS. The ILFPR on $X$ for $S$ is

$$
\bar{R}=\left(\begin{array}{cccc}
{\left[s_{4}, s_{4}\right]} & - & - & {\left[s_{4}, s_{6}\right]} \\
- & {\left[s_{4}, s_{4}\right]} & {\left[s_{3}, s_{4}\right]} & - \\
- & {\left[s_{4}, s_{5}\right]} & {\left[s_{4}, s_{4}\right]} & {\left[s_{3}, s_{5}\right]} \\
{\left[s_{2}, s_{4}\right]} & - & {\left[s_{3}, s_{5}\right]} & {\left[s_{4}, s_{4}\right]}
\end{array}\right) .
$$

Following models (M-3) and (M-4), we derive the complete ILFPR:

$$
\bar{R}=\left(\begin{array}{llll}
{\left[s_{4}, s_{4}\right]} & {\left[s_{5}, s_{5}\right]} & {\left[s_{4}, s_{6}\right]} & {\left[s_{4}, s_{6}\right]} \\
{\left[s_{3}, s_{3}\right]} & {\left[s_{4}, s_{4}\right]} & {\left[s_{3}, s_{4}\right]} & {\left[s_{4}, s_{4}\right]} \\
{\left[s_{2}, s_{4}\right]} & {\left[s_{4}, s_{5}\right]} & {\left[s_{4}, s_{4}\right]} & {\left[s_{3}, s_{5}\right]} \\
{\left[s_{2}, s_{4}\right]} & {\left[s_{4}, s_{4}\right]} & {\left[s_{3}, s_{5}\right]} & {\left[s_{4}, s_{4}\right]}
\end{array}\right) .
$$

With respect to this complete ILFPR, we have $\phi^{*}=4$ following model (M-1), which means that it is inconsistent. Following model (M-2), we derive the following consistent ILFPR:

$$
\bar{R}=\left(\begin{array}{llll}
{\left[s_{4}, s_{4}\right]} & {\left[s_{5}, s_{6}\right]} & {\left[s_{4}, s_{6}\right]} & {\left[s_{4}, s_{6}\right]} \\
{\left[s_{2}, s_{3}\right]} & {\left[s_{4}, s_{4}\right]} & {\left[s_{3}, s_{4}\right]} & {\left[s_{3}, s_{4}\right]} \\
{\left[s_{2}, s_{4}\right]} & {\left[s_{4}, s_{5}\right]} & {\left[s_{4}, s_{4}\right]} & {\left[s_{3}, s_{5}\right]} \\
{\left[s_{2}, s_{4}\right]} & {\left[s_{4}, s_{5}\right]} & {\left[s_{3}, s_{5}\right]} & {\left[s_{4}, s_{4}\right]}
\end{array}\right) .
$$

Notably, $\bar{R}$ is inconsistent following Definition 4 .

\section{GDM with ILFPRs}

This section studies GDM with ILFPRs, which contains two parts. The first part researches the consensus including how to ascertain the DMs' weights and how to increase the consensus level. The second part offers an interactive algorithm for GDM with ILFPRs that can solve inconsistent and incomplete ILFPRs.

For a given GDM problem, Let the DM set be $E=\left\{e_{1}, e_{2}, \ldots, e_{m}\right\}$, and let the object set be $X=\left\{x_{1}, x_{2}, \ldots, x_{n}\right\}$. By $\bar{R}^{k}=\left(\bar{r}_{i j}^{k}\right)_{n \times n}$, we denote the individual ILFPR offered by the DM $e_{k}$ for the predefined LTS $S=\left\{s_{i} \mid i=0,1, \ldots, 2 t\right\}$, where $\bar{r}_{i j}^{k}=\left[r_{i j}^{k, L}, r_{i j}^{k, U}\right]$ for all $i, j=1,2, \ldots, n$ and all $k=1,2, \ldots, m$.

\subsection{Consensus analysis}

To measure the agreement degree of DMs' judgments for the final ranking, researchers usually adopt the consensus measure.

Definition 7. Let $\bar{R}^{k}=\left(\bar{r}_{i j}^{k}\right)_{n \times n}, k=1,2, \ldots, m$, be any $k$ ILFPRs, and let $\bar{R}=\left(\bar{r}_{i j}\right)_{n \times n}$ be the comprehensive ILFPR. The consensus of the ILFPR $\bar{R}^{k}=\left(\bar{r}_{i j}^{k}\right)_{n \times n}$ is defined as: 


$$
G C I\left(\bar{R}^{k}\right)=1-\sum_{i, j=1, i<j}^{n} \frac{1}{2 \operatorname{tn}(n-1)}\left(\left|I\left(r_{i j}^{L}\right)-I\left(r_{i j}^{k, L}\right)\right|+\left|I\left(r_{i j}^{U}\right)-I\left(r_{i j}^{k, U}\right)\right|\right),
$$

where $\bar{r}_{i j}=\oplus_{k=1}^{m} w_{k} \bar{r}_{i j}^{k}$ such that $\bar{r}_{i j}^{k}=\left[r_{i j}^{k, L}, r_{i j}^{k, U}\right]$ and $\bar{r}_{i j}=\left[r_{i j}^{L}, r_{i j}^{U}\right]$ for all $i, j=1,2, \ldots, n$ and all $k=1,2, \ldots, m, t$ is the index of the linguistic term "fair" for the linguistic term set $S=\left\{s_{i} \mid\right.$ $i=0,1, \ldots, 2 t\}$, and $w=\left(w_{1}, w_{2}, \ldots, w_{m}\right)$ is a weight vector such that $\sum_{k=1}^{m} w_{k}=1$ and $w_{k} \geq 0$ for all $k=1,2, \ldots, m$.

Theorem 6. Let $\bar{R}^{k}=\left(\bar{r}_{i j}^{k}\right)_{n \times n}, k=1,2, \ldots, m$, be any $k$ ILFPRs, and let $\bar{R}^{* k}=\left(\bar{r}_{i j}^{* k}\right)_{n \times n}, k=1$, $2, \ldots, m$, be the associated consistent ILFPRs. Then, the comprehensive ILFPR $\bar{R}^{*}=\left(\bar{r}_{i j}^{*}\right)_{n \times n}$ is consistent, where $\bar{r}_{i j}^{*}=\oplus_{k=1}^{m} w_{k} \bar{r}_{i j}^{* k}$ and $w=\left(w_{1}, w_{2}, \ldots, w_{m}\right)$ is a weight vector.

Proof. Following the consistency of each ILFPR $\bar{R}^{* k}$, we derive and

$$
\oplus_{k=1}^{m} w_{k} r_{i j}^{* k, L} \geq\left(\oplus_{k=1}^{m} w_{k} r_{i p}^{* k, L}\right) \oplus\left(\oplus_{k=1}^{m} w_{k} r_{p j}^{* k, L}\right) \ominus s_{t}
$$

$$
\oplus_{k=1}^{m} w_{k} r_{i j}^{* k, U} \leq\left(\oplus_{k=1}^{m} w_{k} r_{i p}^{* k, U}\right) \oplus\left(\oplus_{k=1}^{m} w_{k} r_{p j}^{* k, U}\right) \ominus s_{t}
$$

namely, $r_{i j}^{* L} \geq r_{i p}^{* L} \oplus r_{p j}^{* L} \ominus s_{t}$ and $r_{i j}^{* U} \leq r_{i p}^{* U} \oplus r_{p j}^{* U} \ominus s_{t}$ for each pair of $(i, j)$. Therefore, $\bar{R}^{*}$ is consistent.

To compute the comprehensive ILFPR $\bar{R}=\left(\bar{r}_{i j}\right)_{n \times n}$, the DMs' weights are used. Considering the fact that the higher the individual ILFPR's consensus level is, the bigger the weight of the associated DM will be. Programming model for obtaining the DMs' weights is built as follows:

$$
\begin{aligned}
\psi^{*}= & \min \sum_{k=1}^{m} \sum_{i=1}^{n-1} \sum_{j=i+1}^{n}\left(\eta_{i j}^{k,+}+\eta_{i j}^{k,-}+\mu_{i j}^{k,+}+\mu_{i j}^{k,-}\right) \\
\text { s.t. } & \left\{\begin{array}{l}
\sum_{p=1}^{m} w_{p} I\left(r_{i j}^{p, L}\right)-r_{i j}^{k, L}-\eta_{i j}^{k,+}+\eta_{i j}^{k,-}=0 \\
\sum_{p=1}^{m} w_{p} I\left(r_{i j}^{p, U}\right)-r_{i j}^{k, U}-\mu_{i j}^{k,+}+\mu_{i j}^{k,-}=0 \\
\eta_{i j}^{k,+}, \eta_{i j}^{k,-}, \mu_{i j}^{k,+}, \mu_{i j}^{k,-} \geq 0 \\
i, j=1,2, \ldots, n, i<j, k=1,2, \ldots, m \\
\sum_{p=1}^{m} w_{p}=1, w_{p} \geq 0, p=1,2, \ldots, m \\
w_{p} \in\left[w_{p}^{L}, w_{p}^{U}\right], p=1,2, \ldots, m
\end{array}\right.
\end{aligned}
$$

where the first two constraints are obtained from formula (11) based on the consensus analysis, and $\left[w_{p}^{L}, w_{p}^{U}\right]$ is the given weighting range, $p=1,2, \ldots, m$.

Let $\theta^{*}$ be the offered threshold. If we have $G C I\left(\bar{R}^{k}\right)<\theta^{*}$, namely, the consensus does not meet the requirement, we need to increase $\bar{R}^{k}$ 's consensus level. Considering the fact that the influence of different interval linguistic judgements on consensus is different, the adjustment should not be the same. Furthermore, the adjustment should not change the consistency. On the basis of these analyses, programming model for researching the consensus threshold is built as follows: 


$$
\begin{aligned}
\Delta^{*} & =\max \sum_{i, j=1, i<j}^{n}\left(\alpha_{i j}^{k, L}+\alpha_{i j}^{k, U}\right) \\
s . t . & \left\{\begin{array}{l}
\sum_{i, j=1, i<j}^{n}\left(\left|\sum_{p=1, p \neq k}^{m} w_{p} I\left(r_{i j}^{p, L}\right)+w_{k}\left(\alpha_{i j}^{k, L} I\left(r_{i j}^{k, L}\right)+\left(1-\alpha_{i j}^{k, L}\right) I\left(r_{i j}^{L}\right)\right)-\left(\alpha_{i j}^{k, L} I\left(r_{i j}^{k, L}\right)+\left(1-\alpha_{i j}^{k, L}\right) I\left(r_{i j}^{L}\right)\right)\right|+\right. \\
\left.\left|\sum_{p=1, p \neq k}^{m} w_{p} I\left(r_{i j}^{p, U}\right)+w_{k}\left(\alpha_{i j}^{k, U} I\left(r_{i j}^{k, U}\right)+\left(1-\alpha_{i j}^{k, U}\right) I\left(r_{i j}^{U}\right)\right)-\left(\alpha_{i j}^{k, U} I\left(r_{i j}^{k, U}\right)+\left(1-\alpha_{i j}^{k, U}\right) I\left(r_{i j}^{U}\right)\right)\right|\right)\left(1-\theta^{*}\right) \\
\alpha_{i j}^{k, L} I\left(r_{i j}^{k, L}\right)+\left(1-\alpha_{i j}^{k, L}\right) I\left(r_{i j}^{L}\right) \geq \alpha_{i h}^{k, L} I\left(r_{i h}^{k, L}\right)+\left(1-\alpha_{i h}^{k, L}\right) I\left(r_{i h}^{L}\right)+\alpha_{h j}^{k, L} I\left(r_{h j}^{k, L}\right)+\left(1-\alpha_{h j}^{k, L}\right) I\left(r_{h j}^{L}\right)-t \\
\alpha_{i j}^{k, U} I\left(r_{i j}^{k, U}\right)+\left(1-\alpha_{i j}^{k, U}\right) I\left(r_{i j}^{U}\right) \leq \alpha_{i h}^{k, U} I\left(r_{i h}^{k, U}\right)+\left(1-\alpha_{i h}^{k, U}\right) I\left(r_{i h}^{U}\right)+\alpha_{h j}^{k, U} I\left(r_{h j}^{k, U}\right)+\left(1-\alpha_{h j}^{k, U}\right) I\left(r_{h j}^{U}\right)-t \\
i, h, j=1,2, \ldots, n, h \neq i, j \wedge i<j \\
\alpha_{i j}^{k, L} I\left(r_{i j}^{k, L}\right)+\left(1-\alpha_{i j}^{k, L}\right) I\left(r_{i j}^{L}\right) \leq \alpha_{i j}^{k, U} I\left(r_{i j}^{k, U}\right)+\left(1-\alpha_{i j}^{k, U}\right) I\left(r_{i j}^{U}\right) \\
\alpha_{i j}^{k, L}=\alpha_{j i}^{k, U}, \alpha_{i j}^{k, U}=\alpha_{j i}^{k, L}, i, j=1,2, \ldots, n, i<j
\end{array}\right.
\end{aligned}
$$

where the first constraint is derived from formula (11), the second and third constraints are based on formula (8), the fifth constraint is the condition of ILVs, and the last constraint ensures the corresponding endpoints of ILVs to have the same adjustment.

There are several merits of model (M-6): (1) it permits the left and right endpoints of each ILV to have different adjustments; (2) it ensures the adjusted ILFPRs to satisfy the consensus requirement; (3) it guarantees the adjusted ILFPRs to be still consistent following Definition 6; (4) it ensures the smallest total adjustment under the conditions of the consensus and consistency requirements.

\subsection{An interactive algorithm}

Following the above discussions, this subsection provides an algorithm for GDM with ILFPRs based on the consistency- consensus discussion.

Step 1. Let $\bar{R}^{k}=\left(\bar{r}_{i j}^{k}\right)_{n \times n}$ be the individual ILFPR offered by the DM $e_{k}$ on the object set $X=\left\{x_{1}, x_{2}, \ldots, x_{n}\right\}$ for the predefined LTS $S=\left\{s_{i} \mid i=0,1, \ldots, 2 t\right\}$. If there is no missing value in each individual ILFPR, go to Step 2. If not, models (M-4) and (M-3) are adopted to determine missing linguistic variables;

Step 2. With respect to each completely individual ILFPR $\bar{R}^{k}=\left(\bar{r}_{i j}^{k}\right)_{n \times n}$, model (M-1) is used to judge the consistency. If all individual ILFPRs are consistent, turn to Step 3. If not, model (M-2) is applied to obtain the associated consistent ILFPRs $\bar{R}^{* k}=\left(\bar{r}_{i j}^{* k}\right)_{n \times n}, k=1,2$, ..., $m$;

Step 3. Following individually consistent ILFPRs $\bar{R}^{* k}=\left(\bar{r}_{i j}^{* k}\right)_{n \times n}, k=1,2, \ldots, m$, model (M-5) is adopted to ascertain the weights of DMs, denoted by $w_{k}^{*}, k=1,2, \ldots, m$;

Step 4. We calculate the comprehensively consistent ILFPR $\bar{R}^{*}=\left(\bar{r}_{i j}^{*}\right)_{n \times n}$, where $\bar{r}_{i j}^{*}=\oplus_{k=1}^{m} w_{k}^{*} \bar{r}_{i j}^{* k}, i, j=1,2, \ldots, n$.

Step 5. Formula (11) is used to measure the consensus level of each ILFPR $\bar{R}^{* k}=\left(\bar{r}_{i j}^{* k}\right)_{n \times n}$. Let $\theta^{*}$ be the provided threshold. If we have $G C I\left(\bar{R}^{* k}\right) \geq \theta^{*}$ for all $k=1,2, \ldots, m$, go to Step 6 . If not, model (M-6) is used to research the consensus threshold, and return to Step 4; 
Step 6. Let $\bar{R}^{*}=\left(\bar{r}_{i j}^{*}\right)_{n \times n}$ be the comprehensively consistent ILFPR that satisfies the consensus requirement. We apply the arithmetic average value of each row (Meng et al., 2019) to calculate the interval linguistic priority weight vector, where

$$
\bar{s}=\left(\bar{s}_{1}, \bar{s}_{2}, \ldots, \bar{s}_{n}\right)=\left(\left[s_{1}^{L}, s_{1}^{U}\right],\left[s_{2}^{L}, s_{2}^{U}\right], \ldots,\left[s_{n}^{L}, s_{n}^{U}\right]\right) ;
$$

Step 7. With respect to the interval linguistic priority weights $\overline{s_{i}}, i=1,2, \ldots, n$, we adopt the formula for ranking ILVs (Meng et al., 2016) to $\operatorname{rank} \overline{s_{i}}, \mathrm{i}=1,2, \ldots, \mathrm{n}$, and derive the ranking of objects.

\section{A case study}

This section provides a decision problem about evaluating investment to illustrate the utilization of the new method and to make the comparison.

Example 2 (Meng et al., 2019). An investment company plans to invest some money. Four companies are chosen as possible alternatives: a computer company $x_{1}$, a food company $x_{2}$, a car company $x_{3}$, and a TV company $x_{4}$. Furthermore, four DMs $E=\left\{e_{1}, e_{2}, e_{3}, e_{4}\right\}$ are invited to evaluate them using the predefined discrete LTS: $S=\left\{s_{0}\right.$ : extremely low; $s_{1}$ : very much low; $s_{2}$ : very low; $s_{3}$ : low; $s_{4}$ : a little low; $s_{5}$ : fair; $s_{6}$ : a little high; $s_{7}$ : high; $s_{8}$ : very high; $s_{9}$ : very much high; $s_{10}$ : extremely high\}. Because there are many criteria that influence the judgements of DMs such as the degree of investment risk, the rate of return, and return cycle, it is difficult to require the DMs to offer their exactly qualitative judgements. Thus, the DMs are allowed to employ ILVs to express uncertainty. Suppose that individual ILFPRs are offered as follows:

$$
\begin{aligned}
& \bar{R}^{1}=\left(\begin{array}{llll}
{\left[s_{5}, s_{5}\right]} & {\left[s_{3}, s_{5}\right]} & {\left[s_{5}, s_{7}\right]} & {\left[s_{4}, s_{5}\right]} \\
{\left[s_{5}, s_{7}\right]} & {\left[s_{5}, s_{5}\right]} & {\left[s_{4}, s_{8}\right]} & {\left[s_{6}, s_{7}\right]} \\
{\left[s_{3}, s_{5}\right]} & {\left[s_{2}, s_{6}\right]} & {\left[s_{5}, s_{5}\right]} & {\left[s_{5}, s_{6}\right]} \\
{\left[s_{5}, s_{6}\right]} & {\left[s_{3}, s_{4}\right]} & {\left[s_{4}, s_{5}\right]} & {\left[s_{5}, s_{5}\right]}
\end{array}\right), \bar{R}^{2}=\left(\begin{array}{llll}
{\left[s_{5}, s_{5}\right]} & {\left[s_{5}, s_{7}\right]} & {\left[s_{4}, s_{6}\right]} & {\left[s_{4}, s_{6}\right]} \\
{\left[s_{3}, s_{5}\right]} & {\left[s_{5}, s_{5}\right]} & {\left[s_{4}, s_{6}\right]} & {\left[s_{5}, s_{6}\right]} \\
{\left[s_{4}, s_{6}\right]} & {\left[s_{4}, s_{6}\right]} & {\left[s_{5}, s_{5}\right]} & {\left[s_{5}, s_{7}\right]} \\
{\left[s_{4}, s_{6}\right]} & {\left[s_{4}, s_{5}\right]} & {\left[s_{3}, s_{5}\right]} & {\left[s_{5}, s_{5}\right]}
\end{array}\right),
\end{aligned}
$$

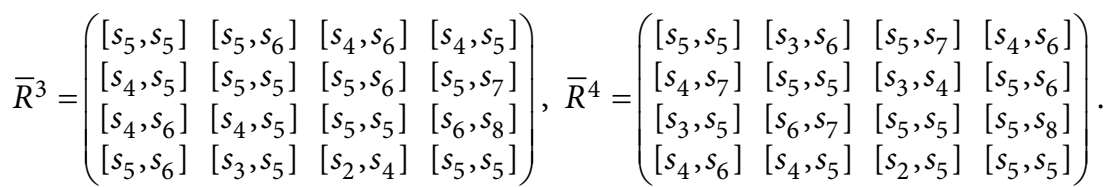

The known weighting range of the DMs is $[0.2,0.4]$. To rank these companies, the procedure is offered as follows:

Step 1. Since all individual ILFPRs are complete, model (M-1) is adopted to judge the consistency. For each of them, following model (M-1) we have $\phi^{*, 1}=7, \phi^{*, 2}=2, \phi^{*, 3}=\phi^{*, 4}=10$. Thus, none of them is consistent based on Definition 6. The consistent ILFPRs based on model (M-2) are

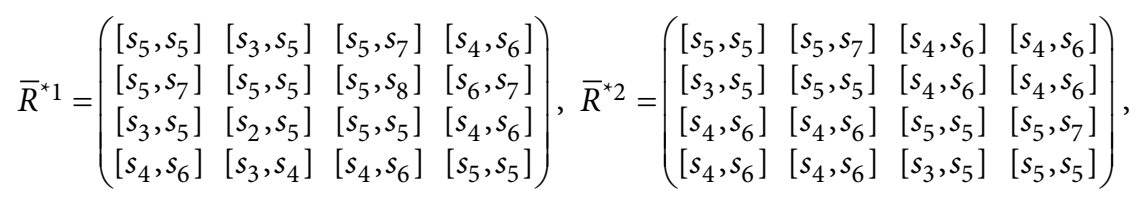




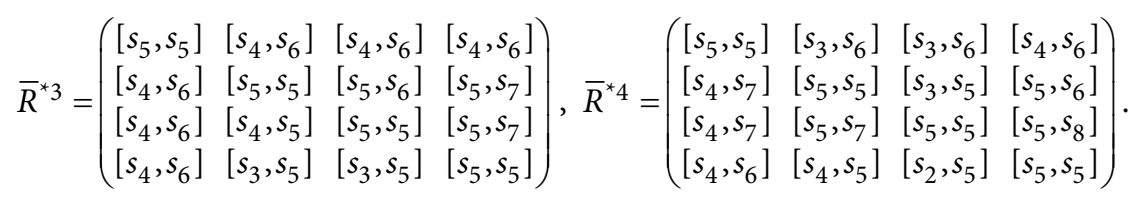

Step 2. Using model (M-5), the DMs' weights are $w_{1}^{*}=w_{2}^{*}=w_{3}^{*}=0.2$ and $w_{4}^{*}=0.4$.

Step 3. Based on the weights of DMs, the comprehensively consistent ILFPR is derived as follows:

$$
\bar{R}^{*}=\left(\begin{array}{cccc}
{\left[s_{5}, s_{5}\right]} & {\left[s_{3.6}, s_{6}\right]} & {\left[s_{3.8}, s_{6.2}\right]} & {\left[s_{4}, s_{6}\right]} \\
{\left[s_{4}, s_{6.4}\right]} & {\left[s_{5}, s_{5}\right]} & {\left[s_{4}, s_{6}\right]} & {\left[s_{5}, s_{6.4}\right]} \\
{\left[s_{3.8}, s_{6.2}\right]} & {\left[s_{4}, s_{6}\right]} & {\left[s_{5}, s_{5}\right]} & {\left[s_{4.8}, s_{7.2}\right]} \\
{\left[s_{4}, s_{6}\right]} & {\left[s_{3.6}, s_{5}\right]} & {\left[s_{2.8}, s_{5.2}\right]} & {\left[s_{5}, s_{5}\right]}
\end{array}\right)
$$

Step 4. Let $\theta^{*}=0.95$, which is the same as the consensus threshold in the literature (Meng et al., 2019). According to formula (11), we obtain

$$
\left\{\begin{array}{l}
G C I\left(\bar{R}^{* 1}\right)=0.915 \\
G C I\left(\bar{R}^{* 2}\right)=0.962 \\
G C I\left(\bar{R}^{* 3}\right)=0.977 \\
G C I\left(\bar{R}^{* 4}\right)=0.958
\end{array}\right.
$$

Because $\operatorname{GCI}\left(\bar{R}^{* 1}\right)<0.95$, we need to improve the consensus level of $\bar{R}^{* 1}$. On the basis of model (M-6), the following adjusted consistent ILFPR is derived:

$$
\bar{R}^{\prime * 1}=\left(\begin{array}{cccc}
{\left[s_{5}, s_{5}\right]} & {\left[s_{3}, s_{5.85}\right]} & {\left[s_{4.15}, s_{7}\right]} & {\left[s_{4}, s_{6}\right]} \\
{\left[s_{4.15}, s_{7}\right]} & {\left[s_{5}, s_{5}\right]} & {\left[s_{5}, s_{6.15}\right]} & {\left[s_{5.15}, s_{7}\right]} \\
{\left[s_{3}, s_{5.85}\right]} & {\left[s_{3.85}, s_{5}\right]} & {\left[s_{5}, s_{5}\right]} & {\left[s_{4}, s_{6.85}\right]} \\
{\left[s_{4}, s_{6}\right]} & {\left[s_{3}, s_{4.85}\right]} & {\left[s_{3.15}, s_{6}\right]} & {\left[s_{5}, s_{5}\right]}
\end{array}\right) .
$$

Furthermore, the associated comprehensively consistent ILFPR is

$$
\bar{R}^{\prime *}=\left(\begin{array}{cccc}
{\left[s_{5}, s_{5}\right]} & {\left[s_{3.6}, s_{6.17}\right]} & {\left[s_{3.63}, s_{6.2}\right]} & {\left[s_{4}, s_{6}\right]} \\
{\left[s_{3.83}, s_{6.4}\right]} & {\left[s_{5}, s_{5}\right]} & {\left[s_{4}, s_{5.63}\right]} & {\left[s_{4.83}, s_{6.4}\right]} \\
{\left[s_{3.8}, s_{6.37}\right]} & {\left[s_{4.37}, s_{6}\right]} & {\left[s_{5}, s_{5}\right]} & {\left[s_{4.8}, s_{7.37}\right]} \\
{\left[s_{4}, s_{6}\right]} & {\left[s_{3.6}, s_{5.17}\right]} & {\left[s_{2.63}, s_{5.2}\right]} & {\left[s_{5}, s_{5}\right]}
\end{array}\right) .
$$

Again using formula (11), we have $\left\{\begin{array}{l}G C I\left(\bar{R}^{\prime * 1}\right)=0.950 \\ G C I\left(\bar{R}^{* 2}\right)=0.958 \\ G C I\left(\bar{R}^{* 3}\right)=0.968 \\ G C I\left(\bar{R}^{* 4}\right)=0.961\end{array}\right.$.

Step 5. Using the row arithmetic average value of $\bar{R}^{{ }^{*}}$, the interval linguistic priority weight vector is

$$
\bar{s}=\left(\left[s_{4.06}, s_{5.84}\right],\left[s_{4.42}, s_{5.86}\right],\left[s_{4.49}, s_{6.18}\right],\left[s_{3.81}, s_{5.34}\right]\right) ;
$$

Step 6. According to the formula for ranking ILVs (Meng et al., 2016), the ranking of objects is $x_{3} \succ x_{2} \succ x_{1} \succ x_{4}$.

Following different methods, ranking orders are derived as shown in Table 1 (Meng et al., 2019). 
Table 1. Ranking orders for different methods

\begin{tabular}{|l|l|}
\hline \multicolumn{1}{|c|}{ Methods } & \multicolumn{1}{c|}{ Ranking orders } \\
\hline Method in (Tapia García et al., 2012) & $x_{1}=x_{2}=x_{3} \succ x_{4}$ \\
\hline Method in (Xu \& Wu, 2013) & $x_{2} \succ x_{3} \succ x_{1} \succ x_{4}$ \\
\hline Method in (Meng et al., 2019) & $x_{3} \succ x_{2} \succ x_{1} \succ x_{4}$ \\
\hline New method & $x_{3} \succ x_{2} \succ x_{1} \succ x_{4}$ \\
\hline
\end{tabular}

Table 1 indicates that different rankings may be derived following different methods. Notably, methods in the literature (Tapia García et al., 2012; Xu \& Wu, 2013) did not consider the consistency of ILFPRs. Although the same ranking is derived following method in the literature (Meng et al., 2019) and the new method, their ranking values are different. The new method based on Definition 6 is more flexible than that offered by Meng et al. (2019) using Definition 4.

\section{Conclusions}

Considering previous research about ILFPRs, this paper defined the constrained interval linguistic arithmetic to study the additive transitivity of ILFPRs, which makes us define consistent ILFPRs in a similar way to ALPRs. Following the defined consistency concept, a new GDM algorithm is provided. The main highlights include: (i) it is more flexible than consistency-based method in the literature (Meng et al., 2019); (ii) it can solve incomplete and inconsistent cases; (iii) programming model for determining the DMs' weights is built; (iv) programming model for researching the consensus threshold is constructed, which endows different ILVs with different adjustments. To show the application and to compare with previous methods, a GDM problem is provided. This paper mainly focuses on the theory and only studies the utilization of the new method in investment problem. Therefore, we will continue to research the application in some other fields including the green supplier selection, evaluation of airline service quality, medical recommendation, and evaluating employees for enterprises. Furthermore, we will continue to research new decision methods with interval linguistic information such as aggregation operator-based method (Zeng, Mu, \& Baležentis, 2017), distance measure-based method (Cheng, Meng, \& Chen, 2017), similarity and entropy based-method (Meng \& Chen, 2016). Furthermore, we shall study other types of preference relations in a similar way to the new method for ILFPRs.

\section{References}

Alonso, S., Cabrerizo, F. J., Chiclana, F., Herrera, F., \& Herrera-Viedma, E. (2009). Group decision making with incomplete fuzzy linguistic preference relations. International Journal of Intelligent Systems, 24(2), 201-222. https://doi.org/10.1002/int.20332

Büyüközkan, G., \& Güleryüz, S. (2014). A new GDM based AHP framework with linguistic interval fuzzy preference relations for renewable energy planning. Journal of Intelligent \& Fuzzy Systems, 27(6), 3181-3195. https://doi.org/10.3233/IFS-141275 
Chen, H. Y., Zhou, L. G., \& Han, B. (2011). On compatibility of uncertain additive linguistic preference relations and its application in the group decision making. Knowledge-Based Systems, 24(6), 816823. https://doi.org/10.1016/j.knosys.2011.03.003

Chen, S. M., \& Lee, L. W. (2012). Autocratic decision making using group recommendations based on the ILLOWA operator and likelihood-based comparison relations. IEEE Transactions on Systems, Man, and Cybernetics-Part A: Systems and Humans, 42(1), 115-129. https://doi.org/10.1109/TSMCA.2011.2157138

Cheng, H., Meng, F. Y., \& Chen, K. (2017). Several generalized interval-valued 2-Tuple linguistic weighted distance measures and their application. International Journal of Fuzzy Systems, 19(4), 967-981. https://doi.org/10.1007/s40815-016-0218-5

Jin, F. F., Ni, Z. W., Pei, L. D., Chen, H. Y., Tao, Z. F., Zhu, X. H., \& Ni, L. P. (2017). Approaches to group decision making with linguistic preference relations based on multiplicative consistency. Computers \& Industrial Engineering, 114, 69-79. https://doi.org/10.1016/j.cie.2017.10.008

Herrera, F., Herrera-Viedma, E., \& Verdegay, J. L. (1996). Direct approach processes in group decision making using linguistic OWA operators. Fuzzy Sets and Systems, 79(2), 75-190.

https://doi.org/10.1016/0165-0114(95)00162-X

Herrera, F., \& Herrera-Viedma, E. (2000). Choice functions and mechanisms for linguistic preference relations. European Journal of Operational Research, 120(1), 144-161.

https://doi.org/10.1016/S0377-2217(98)00383-X

Herrera, F., \& Martinez, L. (2000). A 2-tuple fuzzy linguistic representation model for computing with words. IEEE Transactions on Fuzzy Systems, 8(6), 746-752. https://doi.org/10.1109/91.890332

Herrera-Viedma, E., Martinez, L., Mata, F., \& Chiclana, F. (2005). A consensus support system model for group decision-making problems with multigranular linguistic preference relations. IEEE Transactions on Fuzzy Systems, 13(5), 644-658. https://doi.org/10.1109/TFUZZ.2005.856561

Klir, G. J., \& Yuan, B. (1998). Constrained fuzzy arithmetic: Basic questions and some answers. Soft Computing, 2(2), 100-108. https://doi.org/10.1007/s005000050038

Krejčí, J. (2017). On additive consistency of interval fuzzy preference relations. Computers \& Industrial Engineering, 107, 128-140. https://doi.org/10.1016/j.cie.2017.03.002

Lodwick, W. A., \& Jenkins, O. A. (2013). Constrained intervals and interval spaces. Soft Computing, 17(8), 1393-1402. https://doi.org/10.1007/s00500-013-1006-X

Meng, F. Y., Tan, C. Q., \& Zhang, Q. (2014). An approach to multi-attribute group decision making under uncertain linguistic environment based on the Choquet aggregation operators. Journal of Intelligent \& Fuzzy Systems, 26(2), 769-780. https://doi.org/10.3233/IFS-130767

Meng, F. Y., \& Chen, X. H. (2015). An approach to uncertain linguistic multi-attribute group decision making based on interactive index. International Journal of Uncertainty Fuzziness and KnowledgeBased Systems, 23(3), 319-344. https://doi.org/10.1142/s0218488515500130

Meng, F. Y., Tang, J., \& Xu, Z. S. (2019). Exploiting the priority weights from interval linguistic fuzzy preference relations. Soft Computing, 23(2), 583-597. https://doi.org/10.1007/s00500-017-2878-y

Meng, F. Y., An, Q. X., \& Chen, X. H. (2016). A consistency and consensus-based method to group decision making with interval linguistic preference relations. Journal of the Operational Research Society, 67(11), 1419-1437. https://doi.org/10.1057/jors.2016.28

Meng, F. Y., \& Chen, X. H. (2016). Entropy and similarity measure for Atannasov's interval-valued intuitionistic fuzzy sets and their applications. Fuzzy Optimization and Decision Making, 15(1), 75-101. https://doi.org/10.1007/s10700-015-9215-7

Park, J. H., Gwak, M. G., \& Kwun, Y. C. (2011). Uncertain linguistic harmonic mean operators and their applications to multiple attribute group decision making. Computing, 93(1), 47-64.

https://doi.org/10.1007/s00607-011-0151-2 
Saaty, T. L. (1977). A scaling method for priorities in hierarchical structures. Journal of Mathematical Psychology, 15(3), 234-281. https://doi.org/10.1016/0022-2496(77)90033-5

Tapia García, J. M., del Moral, M. J., Martinez, M. A., \& Herrera-Viedma, E. (2012). A consensus model for group decision making problems with linguistic interval fuzzy preference relations. Expert Systems with Applications, 39(11), 10022-10030. https://doi.org/10.1016/j.eswa.2012.02.008

Tang, J., Meng, F. Y., Li, C. L., \& Li, C. H. (2018). A consistency-based approach to group decision making with uncertain multiplicative linguistic fuzzy preference relations. Journal of Intelligent \& Fuzzy Systems, 35(1), 1037-1054. https://doi.org/10.3233/JIFS-17365

Wang, T. C., \& Chen, Y. H. (2008). Applying fuzzy linguistic preference relations to the improvement of consistency of fuzzy AHP. Information Sciences, 178(19), 3755-3765. https://doi.org/10.1016/j.ins.2008.05.028

$\mathrm{Xu}$, Z. S. (2004a). EOWA and EOWG operators for aggregating linguistic labels based on linguistic preference relations. International Journal of Uncertainty Fuzziness and Knowledge-Based Systems, 12(6), 791-810. https://doi.org/10.1142/S0218488504003211

$\mathrm{Xu}, \mathrm{Z}$. S. (2004b). A method based on linguistic aggregation operators for group decision making with linguistic preference relations. Information Sciences, 166(1-4), 19-30. https://doi.org/10.1016/j.ins.2003.10.006

$\mathrm{Xu}, \mathrm{Z}$. S. (2004c). Uncertain linguistic aggregation operators based approach to multiple attribute group decision making under uncertain linguistic environment. Information Sciences, 168(1-4), 171-184. https://doi.org/10.1016/j.ins.2004.02.003

$\mathrm{Xu}, \mathrm{Z}$. S. (2005). Deviation measures of linguistic preference relations in group decision making. Omega, 33(3), 249-254. https://doi.org/10.1016/j.omega.2004.04.008

$\mathrm{Xu}, \mathrm{Z}$. S. (2006a). Incomplete linguistic preference relations and their fusion. Information Fusion, 7(3), 331-337. https://doi.org/10.1016/j.inffus. 2005.01 .003

$\mathrm{Xu}$, Z. S. (2006b). Induced uncertain linguistic OWA operators applied to group decision making. Information Fusion, 7(2), 231-238. https://doi.org/10.1016/j.inffus.2004.06.005

$\mathrm{Xu}, \mathrm{Z}$. S. (2006c). An approach based on the uncertain LOWG and induced uncertain LOWG operators to group decision making with uncertain multiplicative linguistic preference relations. Decision Support Systems, 41(2), 488-499. https://doi.org/10.1016/j.dss.2004.08.011

$\mathrm{Xu}$, Z. S. (2008). Group decision making based on multiple types of linguistic preference relations. Information Sciences, 178(2), 452-467. https://doi.org/10.1016/j.ins.2007.05.018

$\mathrm{Xu}$, J. P., \& Wu, Z. B. (2013). A maximizing consensus approach for alternative selection based on uncertain linguistic preference relations. Computers \& Industrial Engineering, 64(4), 999-1008. https://doi.org/10.1016/j.cie.2013.01.009

$\mathrm{Xu}$, Y. J., Wu, D., \& Wang, H. M. (2017). A Gower plot-based approach to ascertain and adjust the ordinal and additive inconsistencies for fuzzy linguistic preference relations. International Journal of Fuzzy Systems, 19(6), 2003-2019. https://doi.org/10.1007/s40815-017-0337-7

Zadeh, L. A. (1975). The concept of a linguistic variable and its application to approximate reasoning Part I. Information Sciences, 8(3), 199-249 https://doi.org/10.1016/0020-0255(75)90036-5

Zhou, L. G., He, Y. D., Chen, H. Y., \& Liu, J. P. (2014). On compatibility of uncertain multiplicative linguistic preference relations based on the linguistic COWGA. Applied Intelligence, 40(2), 229-243. https://doi.org/10.1007/s10489-013-0454-4

Zeng, S. Z., Mu, Z. M., \& Baležentis, T. (2017). A novel aggregation method for Pythagorean fuzzy multiple attribute group decision making. International Journal of Intelligent Systems, 40(2), 573-585. https://doi.org/10.1002/int.21953 